\title{
Optimal investment on finite horizon with random discrete order flow in illiquid markets *
}

\author{
Paul Gassiat ${ }^{\dagger}$ Huyên Pham Mihai Sîrbu $^{\S}$
}

October 26, 2018

\begin{abstract}
We study the problem of optimal portfolio selection in an illiquid market with discrete order flow. In this market, bids and offers are not available at any time but trading occurs more frequently near a terminal horizon. The investor can observe and trade the risky asset only at exogenous random times corresponding to the order flow given by an inhomogenous Poisson process. By using a direct dynamic programming approach, we first derive and solve the fixed point dynamic programming equation and then perform a verification argument which provides the existence and characterization of optimal trading strategies. We prove the convergence of the optimal performance, when the deterministic intensity of the order flow approaches infinity at any time, to the optimal expected utility for an investor trading continuously in a perfectly liquid market model with no-short sale constraints.
\end{abstract}

Key words: liquidity modelling, discrete order flow, optimal investment, inhomogenous Poisson process, dynamic programming.

MSC Classification (2000): 60G51, 49K22, 93E20, 91B28.

JEL Classification: G11, C61.

\footnotetext{
${ }^{*}$ H. Pham would like to thank Peter Leoni (Electrabel) for interesting discussions on this illiquidity market modelling.

${ }^{\dagger}$ LPMA, University Paris 7, pgassiat@math.jussieu.fr

${ }^{\ddagger}$ LPMA, University Paris 7, CREST, and Institut Universitaire de France, pham@math.jussieu.fr

$\S$ Department of Mathematics, University of Texas, Austin, sirbu@math.utexas.edu
} 


\section{Introduction}

Financial modeling very often relies on the assumption of continuous-time trading. This is essentially due to the availability of the powerful tool of stochastic integration, which allows for an elegant representation of continuous-time trading strategies, and the analytic tractability of the stochastic calculus, typically illustrated by Itô's formula. Sometimes this assumption may not be very realistic in practice: illiquid markets provide a prime example. Indeed, an important aspect of market liquidity is the time restriction both on trading and observation of the assets. For example, in power markets, trading occurs through a brokered OTC market, and the liquidity is really thin. There could be a possible lack of counterparty for a given order: bids and offers are not available at any time, and may arrive randomly, while the investor can observe the asset only at these arrival times. Moreover, in these markets, because of the physical nature of the underlying asset, trading activity is really low far from the delivery, and is higher near the delivery.

In this paper, we propose a framework that takes into account such liquidity features by considering a discrete order flow. In our model, the investor can observe and trade over a finite horizon only at random times given by an inhomogenous Poisson process encoding the quotes in this illiquid market. To capture the high frequency of trading in the neighborhood of the finite horizon, we assume that the deterministic intensity of this inhomogenous Poisson process approaches infinity as time gets closer to the finite horizon. In this context, the objective of the agent is to maximize his/her expected utility from terminal wealth.

Optimal investment problems with random discrete trading dates were studied by several authors. Rogers and Zane [7] and Matsumoto [5] considered trading times associated to the jump times of a Poisson process, but assumed that price process is observed continuously, so that trading strategies are actually in continuous-time. Recently, Pham and Tankov [6] (see also 2]) investigated an optimal portfolio/consumption choice problem over an infinite horizon, where the asset price, essentially extracted from a Black-Scholes model, can be observed and traded only at the random times corresponding to a Poisson process with constant intensity.

As mentioned above, here we investigate an optimal investment problem over a finite horizon with random trading and observation times given by an inhomogeneous Poisson process with a deterministic intensity going to infinity near the final horizon. The underlying continuous-time asset price is given by an inhomogeneous Lévy process. We use a direct dynamic programming approach for solving this portfolio selection problem. We first derive the fixed point dynamic programming equation (DPE) and provide a constructive proof for the existence of a solution to this DPE in a suitable functional space by means of an iterative procedure. Then, by proving a verification theorem, we obtain the existence and characterization of optimal policies. We also provide an approximation of the optimal strategies that involves only a finite number of iterations. Finally, we address the natural question of convergence of our optimal investment strategy/expected utility when the arrival intensity rate becomes large at all times. We prove that the value function converges to the value function of an agent who can trade continuously in a perfectly liquid mar- 
ket with no-short sale constraints. A related convergence result was recently obtained by Kardaras and Platen [4] by considering continuous-time trading strategies approximated by simple trading strategies with constraints, but with asset prices observed continuously. Here we face some additional subtleties induced by the discrete observation filtrations: the illiquid market investor has less information coming from observing the asset, compared to the continuous-time investor, but he/she has the additional information coming from the arrival times, which is lacking in the perfectly liquid case.

The rest of the paper is organized as follows. Section 2 describes the illiquid market model with the restriction on the trading times, and sets up all the assumptions of the model. We formulate in Section 3 the optimal investment problem, and solve it by a dynamic programming approach and verification argument. Finally, Section 4 is devoted to the convergence issue when the deterministic intensity of arrivals is very large at all times.

\section{The illiquid market model and trading strategies}

We consider an illiquid market in which an investor can trade a risky asset over a finite horizon. In this market, bids and offers are not available at any time, but trading occurs more frequently near the horizon. This is typically the case in power markets with forward contracts. This market illiquidity feature is modelled by assuming that the arrivals of buy/sell orders occur at the jumps of an inhomogeneous Poisson process with an increasing deterministic intensity converging to infinity at the final horizon. In order to obtain an analytically tractable model, we further assume that the discrete-time observed asset prices come from an unobserved continuous-time stochastic process, which is independent of the sequence of arrival times. We may think about the continuous time process as an asset price process based on fundamentals independent of time-illiquidity, which would be actually observed if trading occurred at all times.

More precisely, fixing a probability space $(\Omega, \mathcal{G}, \mathbb{P})$ and a finite horizon $T<\infty$, we consider the fundamental unobserved positive asset price $\left(S_{t}\right)_{0 \leq t \leq T}$. An investor can observe and trade the asset only at some exogenous random times $\left(\tau_{n}\right)_{n \geq 0}, \tau_{0}=0$, such that $\left(\tau_{n}\right)_{n \geq 0}$ and $\left(S_{t}\right)_{0 \leq t \leq T}$ are independent under the physical probability measure $\mathbb{P}$.

In order to obtain a stochastic control problem of Markov type for the utility maximization problem below, we assume an exponential-Lévy structure and some regularity/integrability conditions on the continuous time positive price process $S$. More precisely, we assume that

$$
S_{t}=\mathcal{E}(L)_{t}, \quad 0 \leq t \leq T,
$$

where the process $\left(L_{t}\right)_{0 \leq t \leq T}$ is a semimartingale on $(\Omega, \mathcal{G}, \mathbb{P})$ with independent increments and jumps strictly greater than one. We use $\mathcal{E}(L)$ to denote the Doléans-Dade stochastic exponential of $L$. The assumption $\Delta L>-1$ ensures that the asset $S$, as well as its left-

limit $S_{-}$, are strictly positive at all times. It is well known that a semimartingale with independent increments has deterministic predictable characteristics, see e.g. [3]. Denoting by $\mu(d t, d x, \omega)$ the jump measure and by $\nu(d t, d x)$ its deterministic compensator, we assume 
that the large jumps are integrable, namely that the compensator measure $\nu(d t, d y)$ of $\mu$ satisfies

$$
\int_{0}^{T} \int_{-1}^{\infty} y \nu(d t, d y)<\infty
$$

The Lévy-Khintchin-Itô decomposition implies that

$$
L_{t}=A(t)+M_{t}+\int_{0}^{t} \int_{-1}^{\infty} y(\mu(d t, d y)-\nu(d t, d y)), \quad 0 \leq t \leq T
$$

where $A$ is a deterministic function of bounded variation and $M$ is a continuous local martingale with deterministic quadratic variation, so it is Gaussian. We further assume the following regularity of the deterministic predictable characteristics

1. there exists a function $b:[0, T] \rightarrow \mathbb{R}$ such that

$$
\int_{0}^{T}|b(u)| d u<\infty \text { and } A(t)=\int_{0}^{t} b(u) d u, \quad 0 \leq t \leq T
$$

2. there exists a function $c:[0, T] \rightarrow(0, \infty)$ such that

$$
\int_{0}^{T} c^{2}(u) d u<\infty \text { and }\langle M\rangle_{t}=\int_{0}^{t} c^{2}(u) d u, \quad 0 \leq t \leq T
$$

Conditions (2.1), (2.2) and (2.3) together mean that we actually make the following assumption on $L$ :

(HL)

$$
L_{t}=\int_{0}^{t} b(u) d u+\int_{0}^{t} c(u) d B_{u}+\int_{0}^{t} \int_{-1}^{\infty} y(\mu(d t, d y)-\nu(d t, d y)), \quad 0 \leq t \leq T,
$$

where $\left(B_{t}\right)_{0 \leq T}$ is a Brownian motion on $(\Omega, \mathcal{G}, \mathbb{P})$ independent on the jump measure $\mu$, the measure $\mu$ satisies (2.1) and $b, c$ satisfy (2.2) and (2.3). We denote by

$$
Z_{t, s}=\frac{S_{s}-S_{t}}{S_{t}}=\left\{e^{\left(L_{s}-L_{t}-\frac{1}{2} \int_{t}^{s} c^{2}(u) d u\right)} \prod_{t<u \leq s} e^{-\Delta L_{u}}\left(1+\Delta L_{u}\right)\right\}-1, \quad 0 \leq t \leq s \leq T,
$$

the return between times $t$ and $s$ (if trading is allowed at both times) and denote by

$$
p(t, s, d z)=\mathbb{P}\left[Z_{t, s} \in d z\right]
$$

the distribution of the return.

Remark 2.1 Assumption (HL) ensures that

(i) For all $0 \leq t<s \leq T$ the distribution $p(t, s, d z)$ has support whose interior is equal to $(-1, \infty)$.

(ii) There exists some positive constant $C>0$ such that $\int_{(-1, \infty)}|z| p(t, s, d z) \leq C$, for all $0 \leq t \leq s<T$. This means that, the expectation of the absolute value of the return is 
uniformly bounded in $(t, s)$, i.e. $\mathbb{E}\left[\left|Z_{t, s}\right|\right] \leq C$. The constant $C$ can be chosen as $C=$ $e^{\int_{0}^{T}|b(u)| d u}$, where $b$ is defined in (2.2).

(iii) Since in our model the asset $S$ is not observed at terminal time $T$, there is no loss of generality if we assume that $S_{T}=S_{T-}$, which can be translated in terms of predictable characteristics as $\nu(\{T\},(-1, \infty))=0$. We will make this assumption for the rest of the paper.

The sequence of observation/trading times is represented by the jumps of an inhomogeneous (and independent on $S$ ) Poisson process $\left(N_{t}\right)_{t \in[0, T]}$ with deterministic intensity function $t \in[0, T) \rightarrow \lambda(t) \in(0, \infty)$, such that:

$$
\int_{0}^{t} \lambda(u) d u<\infty,(\forall) 0 \leq t<T \quad \text { and } \quad \int_{0}^{T} \lambda(u) d u=\infty .
$$

The simplest way to actually define such an inhomogeneous Poisson process is to consider a homogeneous Poisson process $M$ with intensity equal to one, independent of $S$ and define

$$
N_{t}=M_{0}^{t} \lambda(u) d u \quad \text { for } \quad 0 \leq t<T .
$$

Condition (2.4) ensures that the probability of having no jumps between any interval $[t, T]$, $t<T$, is null, and so the sequence $\left(\tau_{n}\right)$ converges increasingly to $T$ almost surely when $n$ goes to infinity. We also know that the process of jump times $\left(\tau_{n}\right)_{n \geq 0}$ is a homogeneous Markov chain on $[0, T)$, and its transition probability admits a density given by:

$$
\mathbb{P}\left[\tau_{n+1} \in d s \mid \tau_{n}=t\right]=\lambda(s) e^{-\int_{t}^{s} \lambda(u) d u} 1_{\{t \leq s<T\}} d s,
$$

(which does not depend on $n$ ).

An investor trading in this market can only observe/trade the asset $S$ at the discrete arrival times $\tau_{n}$. Therefore, the only information he/she has is coming from observing the two-dimensional process $\left(\tau_{n}, S_{\tau_{n}}\right)_{n \geq 0}$. Taking this into account, we introduce the discrete observation filtration $\mathbb{F}=\left(\mathcal{F}_{n}\right)_{n \geq 0}$, with $\mathcal{F}_{0}$ trivial and

$$
\mathcal{F}_{n}=\sigma\left\{\left(\tau_{k}, Z_{k}\right): 1 \leq k \leq n\right\}, \quad n \geq 1,
$$

where we denote by

$$
Z_{n}=Z_{\tau_{n-1}, \tau_{n}}, \quad n \geq 1
$$

the observed return process valued in $(-1, \infty)$.

Remark 2.2 Assumption (HL) together with the independence of $S$ and $N$ ensures that for all $n \geq 0$, the (regular) distribution of $\left(\tau_{n+1}, Z_{n+1}\right)$ conditioned on $\mathcal{F}_{n}$ is given as follows:

1. $\mathbb{P}\left[\tau_{n+1} \in d s \mid \mathcal{F}_{n}\right]=\lambda(s) e^{-\int_{\tau_{n}}^{s} \lambda(u) d u} d s$

2. further conditioning on knowing the next arrival time $\tau_{n+1}$, the return $Z_{n+1}$ has distribution

$$
\mathbb{P}\left[Z_{n+1} \in d z \mid \mathcal{F}_{n} \vee \sigma\left(\tau_{n+1}\right)\right]=p\left(\tau_{n}, \tau_{n+1}, d z\right)
$$


In this model a (simple) trading strategy is a real-valued $\mathbb{F}$-adapted process $\alpha=\left(\alpha_{n}\right)_{n \geq 0}$, where $\alpha_{n}$ represents the amount invested in the stock over the period $\left(\tau_{n}, \tau_{n+1}\right]$ after observing the stock price at time $\tau_{n}$. Assuming that the money market pays zero interest rate, the observed wealth process $\left(X_{\tau_{n}}\right)_{n \geq 0}$ associated to a trading strategy $\alpha$ is governed by:

$$
X_{\tau_{n+1}}=X_{\tau_{n}}+\alpha_{n} Z_{n+1}, \quad n \geq 0,
$$

where $X_{0}$ is the initial capital of the investor. In order to simplify notation, we fix once and for all an initial capital $X_{0}>0$ and denote by $\mathcal{A}$ the set of trading strategies $\alpha$ such that the wealth process stays nonnegative:

$$
X_{\tau_{n}} \geq 0, \quad n \geq 1
$$

For the rest of the paper, we will call simple these trading strategies where trading occurs only at the discrete times $\tau_{n}, n \geq 0$.

Remark 2.3 Constrained strategies. From (2.8) and the support property of $Z_{t, s}$ in Remark 2.1(i), the admissibility condition (2.9) on $\alpha \in \mathcal{A}$ means that we have a no-short sale constraint (on both the risky and savings account asset):

$$
0 \leq \alpha_{n} \leq X_{\tau_{n}}, \text { for all } n \geq 0 .
$$

Moreover, since $Z_{n}>-1$ a.s. for all $n \geq 1$, the wealth process associated to $\alpha \in \mathcal{A}_{0}$ is actually strictly positive:

$$
X_{\tau_{n}}>0, \quad n \geq 0
$$

For technical reasons, some related to the asymptotic behavior in Section 4.1, we need to define some continuous time filtrations along with the discrete filtration $\mathbb{F}$. To avoid confusion, we will denote by $\mathbb{G}$ (with different parameters) all continuous-time filtrations. In this spirit, we define the filtration $\mathbb{G}=\left(\mathcal{G}_{t}\right)_{0 \leq t \leq T}$ generated by observing continously the process $S$ and the arrival times as

$$
\mathcal{G}_{t}=\sigma\left\{\left(S_{u}, N_{u}\right), 0 \leq u \leq t\right\} \vee \mathcal{N}, \quad 0 \leq t \leq T,
$$

where $N$ is the inhomogenous Poisson process in (2.5) and $\mathcal{N}$ are all the null sets of $\mathcal{G}$ under the historical measure $\mathbb{P}$. We would like to point out that, because of the Lévy structure of the joint process $(S, N)$, the filtration $\mathbb{G}$ is right continous, so it satisfies the usual conditions. In addition, we have the strict inclusion:

$$
\mathcal{F}_{n} \subset \mathcal{G}_{\tau_{n}} \text { for all } n \geq 1
$$

We make an additional assumption on the model, which, among others, precludes arbitrage possibilities:

(NA)

$$
\int_{0}^{T} \frac{b^{2}(u)}{c^{2}(u)} d u<\infty
$$


Remark 2.4 Underssumption (NA), we can define the probability measure $\mathbb{Q}$ by

$$
\frac{d \mathbb{Q}}{d \mathbb{P}}=e^{-\int_{0}^{T} \frac{b(u)}{c(u)} d W_{u}-\int_{0}^{T}\left(\frac{b(u)}{c(u)}\right)^{2} d u} .
$$

Under $\mathbb{Q}$, the process $N$ has the same law as under $\mathbb{P}$, and $\left(\tau_{n}\right)_{n \geq 0}$ and $\left(S_{t}\right)_{0 \leq t \leq T}$ are still independent under $\mathbb{Q}$. Moreover, the process $S$ is a positive $(\mathbb{Q}, \mathbb{G})$-local martingale so a supermartingale. This means that the discrete-time process $\left(S_{\tau_{n}}\right)_{n \geq 0}$ is a $(\mathbb{Q}, \mathbb{F})$ supermartingale as well.

Remark 2.5 Embedding in a continuous-time wealth process. Given $\alpha \in \mathcal{A}$ with corresponding wealth process $\left(X_{\tau_{n}}\right)_{n}$ in (2.8), let us define the continuous time process $\left(X_{t}\right)_{0 \leq t<T}$ by

$$
\begin{aligned}
X_{t} & =X_{\tau_{n}}+\alpha_{n} Z_{\tau_{n}, t}, \quad \tau_{n}<t \leq \tau_{n+1}, \quad n \geq 0, \\
& =X_{0}+\int_{0}^{t} H_{u} d S_{u}, \quad 0 \leq t<T,
\end{aligned}
$$

where $H$ is the simple and $\mathbb{G}$-predictable process

$$
H_{t}=\sum_{n=0}^{\infty} \frac{\alpha_{n}}{S_{\tau_{n}}} \mathbb{I}_{\left\{\tau_{n}<t \leq \tau_{n+1}\right\}}, \quad 0 \leq t<T,
$$

representing the number of shares invested in the risky asset. From (2.10) and since $S_{t}>$ 0 , so $Z_{\tau_{n}, t}>-1, n \geq 0$, we notice that the continuous time process $X$ is strictly positive: $X_{t}>0$ for $0 \leq t<T$. Moreover, according to Remark 2.4. $\left(X_{t}\right)_{0 \leq t<T}$ is a $(\mathbb{Q}, \mathbb{G})$-local martingale, hence a super-martingale up to $T$. Consequently, we also have $X_{t-}>0$ for $0 \leq t<T$. We would like to point out that the definition of $X_{\tau_{n}}$ in (2.8) is consistent with (2.12), so $\left(X_{\tau_{n}}\right)_{n \geq 0}$ is a positive $\mathbb{F}$-supermartingale under $\mathbb{Q}$. Therefore, for each $\alpha \in \mathcal{A}$ we may define the terminal wealth value by:

$$
X_{T}=\lim _{n \rightarrow \infty} X_{\tau_{n}}=\lim _{t \nearrow T} X_{t}=X_{0}+\sum_{n=0}^{\infty} \alpha_{n} Z_{n+1}
$$

and, since $S_{T}=S_{T-}$ we also have

$$
X_{T}=X_{0}+\int_{0}^{T} H_{u} d S_{u}
$$

where the integrand $H$ is related to the simple trading strategy $\alpha$ as described above. The supermartingale property implies the budget constraint

$$
\mathbb{E}^{\mathbb{Q}}\left[X_{T}\right] \leq X_{0}
$$

The continuous time wealth process $X$ has the meaning of a shadow wealth process: it is not observed except for at times $\tau_{n}, n \geq 0$. The no-short sale constraints (2.10) is translated in terms of the number of shares held as

$$
0 \leq H_{t} S_{t-} \leq X_{t-}, \quad 0 \leq t<T \text {. }
$$


We denote by $\mathcal{X}$ the set of all wealth processes $\left(X_{t}\right)_{0 \leq t \leq T}$ given by (2.12), by using simple trading strategies under the no-short sale constraint (2.10)/(2.13). We denote by $\overline{\mathcal{X}}$ the set of all positive wealth processes $\left(X_{t}\right)_{0 \leq t \leq T}$ given by (2.12), by using general $\mathbb{G}$ predictable and $S$-integrable processes $H$ satisfying (2.13). We clearly have $\mathcal{X} \subset \overline{\mathcal{X}}$.

For technical reasons, it is sometimes convenient to regard trading strategies equivalently in terms of proportions of wealth. For any continuous time wealth process $X \in$ $\overline{\mathcal{X}}$ associated to a trading strategy $H$ satisfying (2.13), let us consider the process $\pi=$ $\left(\pi_{t}\right)_{0 \leq t \leq T}$, defined by: $\pi_{t}=H_{t} S_{t-} / X_{t-}$, and notice that $\pi$ is valued in $[0,1]$ by (2.13). We stress the dependence of the wealth on the proportion $\pi$, and denote by $X^{(\pi)}=X$, which is then written in a multiplicative way as

$$
X_{.}^{(\pi)}=X_{0} \mathcal{E}\left(\int_{0}^{\cdot} \pi \frac{d S}{S_{-}}\right)=X_{0} \mathcal{E}\left(\int_{0}^{\cdot} \pi d L\right),
$$

where $\mathcal{E}$ is the Doléans-Dade operator. Denote by $\mathcal{D}(\mathbb{G})$ the set of all $\mathbb{G}$-predictable processes $\pi$ valued in $[0,1]$. It is then clear that

$$
\overline{\mathcal{X}}=\left\{X^{(\pi)} \mid \pi \in \mathcal{D}(\mathbb{G})\right\} .
$$

\section{Optimal investment problem and dynamic programming}

We investigate an optimal investment problem in the illiquid market described in the previous section. Let us consider an utility function $U$ defined on $(0, \infty)$, strictly increasing, strictly concave and $C^{1}$ on $(0, \infty)$, and satisfying the Inada conditions: $U^{\prime}\left(0^{+}\right)=\infty, U^{\prime}(\infty)$ $=0$. We make the following additional assumptions on the utility function $U$ :

(HU) (i) there exist some constants $C>0$ and $p \in(0,1)$ such that

$$
U^{+}(x) \leq C\left(1+x^{p}\right), \quad(\forall) x>0
$$

where $U^{+}=\max (U, 0)$

(ii) Either $U(0)>-\infty$, or $U(0)=-\infty$ and there exist some constants $C^{\prime}>0$ and $p^{\prime}<0$ such that

$$
U^{-}(x) \leq C\left(1+x^{p^{\prime}}\right), \quad(\forall) x>0
$$

where $U^{-}=\max (-U, 0)$.

The above assumptions include most popular utility functions, in particular those with constant relative risk aversion $1-p>0$, in the form $U(x)=\left(x^{p}-1\right) / p, x>0$.

Given the chosen positive initial wealth $X_{0}>0$, we consider the optimal investment problem:

$$
V_{0}=\sup _{\alpha \in \mathcal{A}} \mathbb{E}\left[U\left(X_{T}\right)\right]=\sup _{X \in \mathcal{X}} \mathbb{E}\left[U\left(X_{T}\right)\right] .
$$

Our aim is to provide an analytic solution to the control problem (3.1) using direct dynamic programming, i.e. first solve the Dynamic Programming Equation (DPE) analytically and then perform a verification argument. Therefore, there is no need to either define the value function at later times or to prove the Dynamic Programming Principle (DPP).

The Lemma below provides the intuition behind the (DPE): 
Lemma 3.1 Assume (HL) holds true. Let $\alpha \in \mathcal{A}$ and let $\left(X_{\tau_{n}}\right)_{n \geq 0}$ be the wealth process associated with the trading strategy $\alpha$. Consider a measurable function $v:[0, T) \times(0, \infty) \rightarrow$ $\mathbb{R}$. For a fixed $n \geq 0$, if $v\left(\tau_{n+1}, X_{\tau_{n+1}}\right) \in L^{1}(\Omega, \mathcal{G}, \mathbb{P})$, then

$$
\mathbb{E}\left[v\left(\tau_{n+1}, X_{\tau_{n+1}}\right) \mid \mathcal{F}_{n}\right]=\int_{\tau_{n}}^{T} \int_{(-1, \infty)} \lambda(s) e^{-\int_{\tau_{n}}^{s} \lambda(u) d u} v\left(s, X_{\tau_{n}}+\alpha_{n} z\right) p\left(\tau_{n}, s, d z\right) d s,
$$

where the above equality holds $\mathbb{P}$-a.s.

Proof. From Remark 2.2 we have

$$
\begin{aligned}
\mathbb{E}\left[v\left(\tau_{n+1}, X_{\tau_{n+1}}\right) \mid \mathcal{F}_{n}\right] & =\mathbb{E}\left[\mathbb{E}\left[v\left(\tau_{n+1}, X_{\tau_{n}}+\alpha_{n} Z_{n+1}\right) \mid \mathcal{F}_{n} \vee \sigma\left(\tau_{n+1}\right)\right] \mid \mathcal{F}_{n}\right] \\
& =\mathbb{E}\left[\int_{(-1, \infty)} v\left(\tau_{n+1}, X_{\tau_{n}}+\alpha_{n} z\right) p\left(\tau_{n}, \tau_{n+1}, d z\right) \mid \mathcal{F}_{n}\right] \\
& =\int_{\tau_{n}}^{T} \int_{(-1, \infty)} \lambda(s) e^{-\int_{\tau_{n}}^{s} \lambda(u) d u} v\left(s, X_{\tau_{n}}+\alpha_{n} z\right) p\left(\tau_{n}, s, d z\right) d s
\end{aligned}
$$

Taking into account the above Lemma, we can now formally write down the Dynamic Programming Equation as

$$
\begin{aligned}
v(t, x) & =\sup _{a \in[0, x]} \int_{t}^{T} \int_{(-1, \infty)} \lambda(s) e^{-\int_{t}^{s} \lambda(u) d u} v(s, x+a z) p(t, s, d z) d s \\
& =\sup _{\pi \in[0,1]} \int_{t}^{T} \int_{(-1, \infty)} \lambda(s) e^{-\int_{t}^{s} \lambda(u) d u} v(s, x(1+\pi z)) p(t, s, d z) d s
\end{aligned}
$$

for all $(t, x) \in[0, T) \times(0, \infty)$ together with the natural terminal condition

$$
\lim _{t \nearrow T, x^{\prime} \rightarrow x} v\left(t, x^{\prime}\right)=U(x), \quad x>0 .
$$

It appears that the right space of functions to be looking for a solution of (3.2)-(3.3) is actually the space $\mathcal{C}_{U}([0, T) \times(0, \infty))$ of measurable functions $w$ on $[0, T) \times(0, \infty)$, such that

1. $w(t,$.$) is concave on (0, \infty)$ for all $t \in[0, T)$, and

2. for some $C=C(w)>0$, we have

$$
U(x) \leq w(t, x) \leq C(1+x), \quad \forall(t, x) \in[0, T) \times(0, \infty) .
$$

For any $w \in \mathcal{C}_{U}([0, T) \times(0, \infty))$, we consider the measurable function $\mathcal{L} w$ on $[0, T) \times(0, \infty)$ defined by:

$$
\mathcal{L} w(t, x)=\sup _{\pi \in[0,1]} \int_{t}^{T} \int_{(-1, \infty)} \lambda(s) e^{-\int_{t}^{s} \lambda(u) d u} w(s, x(1+\pi z)) p(t, s, d z) d s .
$$

Lemma 3.2 below shows that the operator

$$
\mathcal{L}: \mathcal{C}_{U}([0, T) \times(0, \infty)) \rightarrow \mathcal{C}_{U}([0, T) \times(0, \infty)),
$$


is well defined. Therefore, we are looking for a a solution $w \in \mathcal{C}_{U}([0, T) \times(0, \infty))$ to the DPE:

$$
\left\{\begin{array}{l}
\mathcal{L} w=w \\
\lim _{t \nearrow T, x^{\prime} \rightarrow x} w\left(t, x^{\prime}\right)=U(x) .
\end{array}\right.
$$

In order to solve the DPE and perform the verification argument, we need some technical details collected in the subsection below:

\subsection{A supersolution of the DPE and other technical details}

Lemma 3.2 Assume that (HL) holds. For any $w \in \mathcal{C}_{U}([0, T) \times(0, \infty)), \mathcal{L} w$ also belongs to $\mathcal{C}_{U}([0, T) \times(0, \infty))$. For each $(t, x) \in[0, T) \times(0, \infty)$ the supremum in (3.5) is attained at some $\pi(t, x)$ which can be chosen measurable in $(t, x)$.

Proof. Given $w \in \mathcal{C}_{U}([0, T) \times(0, \infty))$, let us consider the measurable function $\widehat{w}$ defined on $[0, T) \times(0, \infty) \times[0,1]$ by:

$$
\widehat{w}(t, x, \pi)=\int_{t}^{T} \int_{(-1, \infty)} \lambda(s) e^{-\int_{t}^{s} \lambda(u) d u} w(s, x(1+\pi z)) p(t, s, d z) d s,
$$

so that $\mathcal{L} w(t, x)=\sup _{\pi \in[0,1]} \widehat{w}(t, x, \pi)$. Observe from (3.4) and the integrability condition in Remark 2.1 (ii) that $\hat{w}$ is well-defined on $[0, T) \times(0, \infty) \times[0,1]$ and satisfies:

$$
-\infty \leq \widehat{w}(t, x, \pi) \leq C(1+x), \quad \forall(t, x, \pi) \in[0, T) \times(0, \infty) \times[0,1],
$$

for some positive constant $C>0$ and, by (3.4), for each $(t, x) \in[0, T) \times(0, \infty)$, we have

$$
\widehat{w}(t, x, 0) \geq U(x) .
$$

As a matter of fact, one can easily see that $\widehat{w}(t, x, \pi)$ is actually finite for any $\pi \in[0,1)$ and may only equal negative infinity for $\pi=1$. Consequently, for fixed $(t, x) \in[0, T) \times(0, \infty)$, $\widehat{w}(t, x,$.$) is a proper one-dimensional concave function defined on [0,1]$ (concavity follows easily from that of $w$ ). In addition, using the linear growth (3.4) together with Fatou lemma, we obtain that $\pi \in[0,1] \rightarrow \widehat{w}(t, x, \pi)$ is upper semicontinuous (this refers to the endpoints $\pi=0,1$ since the function is continuous on $(0,1)$ being finite and concave). Therefore $\mathcal{L} w(t, x)=\max _{\pi \in[0,1]} \widehat{w}(t, x, \pi)$, where the maximum is attained at some $\pi=\pi(t, x)$ which can be chosen measurable in $(t, x)$, see e.g. Ch. 11 in [1]. In addition, since $\pi \rightarrow \widehat{w}(t, x, \pi)$ is continuous on $(0,1)$, the function $\mathcal{L} w$ has the additional representation

$$
\mathcal{L} w(t, x)=\sup _{\pi \in[0,1] \cap \mathbb{Q}} \widehat{w}(t, x, \pi),
$$

which shows that $\mathcal{L} w$ is measurable. The concavity of $w(t,$.$) implies the concavity of (x, a)$ $\in\{(x, a) \in(0, \infty) \times \mathbb{R}: a \in[0, x]\} \rightarrow \widehat{w}(t, x, a / x)$ for all $t \in[0, T)$. This easily implies that $\mathcal{L} w(t,$.$) is also concave on (0, \infty)$ for all $t \in[0, T)$. Finally, it is clear from (3.7) and (3.8) that $\mathcal{L} w$ satisfies also the growth condition:

$$
U(x) \leq \mathcal{L} w(t, x) \leq C(1+x), \quad \forall(t, x) \in[0, T) \times(0, \infty) .
$$

The next lemma constucts a supersolution $f \in C_{U}([0, T) \times(0, \infty))$ for the DPE: 
Lemma 3.3 Assume that (HL), (NA) and (HU) hold. Define

$$
f(t, x)=\inf _{y>0}\left\{\mathbb{E}\left[\tilde{U}\left(y Y_{t, T}\right)\right]+y x\right\} \quad(t, x) \in[0, T] \times(0, \infty),
$$

where

$$
Y_{t, T}=e^{-\int_{t}^{T} \frac{b(u)}{c(u)} d W_{u}-\int_{t}^{T}\left(\frac{b(u)}{c(u)}\right)^{2} d u}
$$

and $\tilde{U}$ is the Fenchel-Legendre transform of $U$ :

$$
\tilde{U}(y)=\sup _{x>0}[U(x)-x y]<\infty, \quad \forall y>0 .
$$

Then, $f$ lies in the set $\mathcal{C}_{U}([0, T) \times(0, \infty))$, and satisfies

$$
\left\{\begin{array}{l}
\mathcal{L} f \leq f \\
\lim _{t \nearrow T, x^{\prime} \rightarrow x} f\left(t, x^{\prime}\right)=U(x) .
\end{array}\right.
$$

Proof. Jensen's inequality gives $\mathbb{E}\left[\tilde{U}\left(y Y_{t, T}\right)\right] \geq \tilde{U}(y)$, so

$$
f(t, x) \geq \inf _{y>0}\{\tilde{U}(y)+y x\}=U(x) .
$$

From the definition of $f$ we know that

$$
f(t, x) \leq \mathbb{E}\left[\tilde{U}\left(y Y_{t, T}\right)\right]+y x, \quad(\forall) y>0 .
$$

Fix a $y_{0}>0$. Jensen's inequality together with Assumption (HU)(i) shows that

$$
\mathbb{E}\left[\tilde{U}\left(y_{0} Y_{t, T}\right)\right] \leq \mathbb{E}\left[\tilde{U}\left(y_{0} Y_{0, T}\right)\right]<\infty
$$

so

$$
f(t, x) \leq \mathbb{E}\left[\tilde{U}\left(y_{0} Y_{t, T}\right)\right]+y_{0} x \leq C(1+x) \quad(\forall)(t, x) \in[0, T] \times(0, \infty) .
$$

This shows that $f \in \mathcal{C}_{U}([0, T) \times(0, \infty))$. Using assumption (HU) (both (i) and (ii)) and the expression (3.9) we see that

$$
\lim _{t \nearrow T} \mathbb{E}\left[\tilde{U}\left(y Y_{t, T}\right)\right]=\tilde{U}(y),(\forall) y>0 .
$$

We can now use this in (3.12) to deduce that

$$
U(x) \leq \liminf _{t \nearrow T, x^{\prime} \rightarrow x} f\left(t, x^{\prime}\right) \leq \limsup _{t \nearrow T, x^{\prime} \rightarrow x} f\left(t, x^{\prime}\right) \leq \tilde{U}(y)+x y,(\forall) y>0 .
$$

Taking the infimum over $y$ we obtain the terminal condition. For each fixed $t$, the function $f(t, \cdot)$ is finite and concave on $(0, \infty)$, so the only thing left to check is the supersolution property. Fix $0 \leq t \leq s \leq T$ and $x>0$. Denote by $h(z)=\mathbb{E}\left[\tilde{U}\left(z Y_{s, T}\right)\right]$ and fix $y>0$ and $\pi \in[0,1]$. By the very definition of the function $f$ we have that

$$
f\left(s, x\left(1+\pi Z_{t, s}\right)\right) \leq h\left(y Y_{t, s}\right)+x\left(1+\pi Z_{t, s}\right) y Y_{t, s} .
$$


Using independence and the definition of $h$, we obtain

$$
\begin{aligned}
\mathbb{E}\left[f\left(s, x\left(1+\pi Z_{t, s}\right)\right)\right] & \leq \mathbb{E}\left[h\left(y Y_{t, s}\right)+x\left(1+\pi Z_{t, s}\right) y Y_{t, s}\right]= \\
& =\mathbb{E}\left[\tilde{U}\left(y Y_{t, s} Y_{s, T}\right)\right]+\mathbb{E}\left[x\left(1+\pi Z_{t, s}\right) y Y_{t, s}\right] \leq \mathbb{E}\left[\tilde{U}\left(y Y_{t, T}\right)\right]+x y .
\end{aligned}
$$

Taking the inf over all $y$ and recalling the definition of $f(t, x)$ we obtain

$$
f(t, x) \geq \mathbb{E}\left[f\left(s, x\left(1+\pi Z_{t, s}\right)\right)\right]=\int_{(-1, \infty)} f(s, x(1+\pi z)) p(t, s, d z)
$$

for all $\pi$ and $s$. For a fixed $\pi$, we can integrate over $s$ to obtain

$$
f(t, x) \geq \int_{t}^{T} \int_{(-1, \infty)} \lambda(s) e^{-\int_{t}^{s} \lambda(u) d u} f(s, x(1+\pi z)) p(t, s, d z) d s,
$$

and then taking the supremum over $\pi$ we obtain $f(t, x) \geq(\mathcal{L} f)(t, x)$, so the proof is complete. We would like to point out that, due to the linear growth condition on $f$, as well as Remark 2.1 (ii), all expectations/integrals above are well defined, but may be negative infinity. In other words, the positive parts in all expectations/integrals are actually integrable.

Remark 3.1 We would like to point out that the whole analysis in this paper extends to the case when the Brownian part of the process $L$ is degenerate, as long as the jumps have full support on $(-1, \infty)$ and the jump measure allows for a martingale measure with density process $Y$ that can replace the definition (3.9) in the corresponding proofs. In other words, the assumptions (HL) and (NA) can be relaxed to include the situation when the drift can be removed by changing the jump measure appropriately, if the Gaussian part is missing.

It turns out that, for the verification arguments below, we also need an assumption on the integrability of jumps.

(HI): (i) there exists $q>1$ such that

$$
\int_{0}^{T} \int_{0}^{\infty}\left((1+y)^{q}-1-q y\right) \nu(d t, d y)<\infty
$$

(ii) If the utility function $U$ satisfies $U(0)=-\infty$, then there exists $r<p^{\prime}<0$ (where $p^{\prime}$ is given in $(\mathbf{H U})(\mathrm{ii}))$ such that

$$
\int_{0}^{T} \int_{-1}^{0}\left((1+y)^{r}-1-r y\right) \nu(d t, d y)<\infty .
$$

(iii) there are no predictable jumps, i.e. $\nu(\{t\},(-1, \infty))=0$ for each $t$

Remark 3.2 Using convexity, it is an easy exercise to see that assumption (HI) (i) can actually be rephrased as $\nu_{q}([0, T])<\infty$, and assumption (HI)(ii) as $\nu_{r}([0, T])<\infty$ where

$$
\nu_{l}(d t)=\int_{-1}^{\infty} \sup _{\pi \in[0,1]}\left((1+\pi y)^{l}-1-l \pi y\right) \nu(d t, d y)
$$


We now prove a crucial uniform integrability condition, but before that we denote by $\mathcal{T}$ the set of random times $0 \leq \tau<T$ which are stopping times with respect to the filtration $\mathbb{G}$.

Lemma 3.4 Assume that (HL), (HU), (NA) and (HI) hold.

(1) For any $X \in \overline{\mathcal{X}}$, the family $\left(f^{+}\left(\tau, X_{\tau}\right)\right)_{\tau \in \mathcal{T}}$ is uniformly $\mathbb{P}$-integrable.

(2) For any $X \in \overline{\mathcal{X}}$, the family $\left(U^{-}\left(X_{\tau}\right)\right)_{\tau \in \mathcal{T}}$, is uniformly $\mathbb{P}$-integrable.

Proof. Assume $\nu_{l}([0, T])<\infty$ for some $l$. Consider $X=X^{(\pi)} \in \overline{\mathcal{X}}$ for some $\pi \in \mathcal{D}(\mathbb{G})$, and recall that

$$
d X_{t}^{(\pi)}=\pi_{t} X_{t-}^{(\pi)} d L_{t}, \quad 0 \leq t \leq T .
$$

In order to simplify notation, we supress the upper indices of $X$. We apply Itô formula to $\left(X_{t}\right)^{l}$ to conclude that

$$
\begin{array}{r}
X_{t}^{l}=x^{l}+\int_{0}^{t}\left(X_{u-}\right)^{l}\left(l \pi_{u} b(u)+l(l-1) c^{2}(u) \pi^{2}(u)\right) d u+ \\
\int_{0}^{t} \int_{-1}^{\infty}\left(X_{u-}\right)^{l}\left(\left(1+\pi_{u} y\right)^{l}-1-l \pi_{u} y\right) \nu(d u, d y)+{ }^{\prime \prime} \text { local martingale }{ }^{\prime \prime}
\end{array}
$$

Fix a stopping time $\tau \in \mathcal{T}$. If $T_{n}^{\prime}$ is a sequence of localizing stopping times for the local martingale part, denote by $T_{n}=T_{n}^{\prime} \wedge\left\{\inf t:\left(X_{t}\right)^{l} \geq n\right\}$. Observe that $T_{n} \nearrow T$ a.s. since $X_{-}$is locally bounded and locally bounded away from zero. We then have, for each $0 \leq t<T$,

$$
\begin{aligned}
\mathbb{E}\left[\left(X_{t \wedge \tau \wedge T_{n}}\right)^{l}\right]=x^{l} & +\mathbb{E}\left[\int _ { 0 } ^ { t \wedge \tau \wedge T _ { n } } ( X _ { u - } ) ^ { l } \left\{\left(l \pi_{u} b(u)+l(l-1) c^{2}(u) \pi^{2}(u)\right) d u\right.\right. \\
& \left.\left.+\int_{-1}^{\infty}\left(\left(1+\pi_{u} y\right)^{l}-1-l \pi_{u} y\right) \nu(d u, d y)\right\}\right] \\
\leq x^{l}+ & \mathbb{E}\left[\int_{0}^{t \wedge \tau \wedge T_{n}}\left(X_{u-}\right)^{l}\left\{\left(|l b(u)|+\left|l(l-1) c^{2}(u)\right|\right) d u+\nu_{l}(d u)\right\}\right]
\end{aligned}
$$

Since $\left(X_{u-}\right)^{l} \leq n$ for $0 \leq u \leq \tau \wedge T_{n}$ and $\nu_{l}([0, T])<\infty$, we conclude that

$$
\mathbb{E}\left[\left(X_{t \wedge \tau \wedge T_{n}}\right)^{l}\right]<\infty, \quad 0 \leq t<T .
$$

In addition, since the paths of the process $X^{l}$ are RCLL and $\nu_{l}(\{u\})=0$ for each $0 \leq u \leq T$ (because of (HI) part (iii)), we have that, for each $0 \leq t<T$, with $\mathbb{P}$-probability one

$$
\begin{array}{r}
\int_{0}^{t \wedge \tau \wedge T_{n}}\left(X_{u-}\right)^{l}\left\{\left(|l b(u)|+\left|l(l-1) c^{2}(u)\right|\right) d u+\nu_{l}(d u)\right\}= \\
\int_{0}^{t \wedge \tau \wedge T_{n}}\left(X_{u}\right)^{l}\left\{\left(|l b(u)|+\left|l(l-1) c^{2}(u)\right|\right) d u+\nu_{l}(d u)\right\} \leq \\
\int_{0}^{t}\left(X_{u \wedge \tau \wedge T_{n}}\right)^{l}\left\{\left(|l b(u)|+\left|l(l-1) c^{2}(u)\right|\right) d u+\nu_{l}(d u)\right\} .
\end{array}
$$

Replacing this in (3.14) and using Fubini, we obtain

$$
\mathbb{E}\left[\left(X_{t \wedge \tau \wedge T_{n}}\right)^{l}\right] \leq x^{l}+\int_{0}^{t} \mathbb{E}\left[\left(X_{u \wedge \tau \wedge T_{n}}\right)^{l}\right]\left\{\left(|l b(u)|+\left|l(l-1) c^{2}(u)\right|\right) d u+\nu_{l}(d u)\right\} .
$$


Now, using (3.15) and $\nu_{l}([0, T])<\infty$, we can apply Gronwall in (3.16) to conclude that $\mathbb{E}\left[\left(X_{t \wedge \tau \wedge T_{n}}\right)^{l}\right] \leq M(l)<\infty$, for each $0 \leq t<T$, where $M(l)$ does not depend on $\tau$ or $n$. Letting $n \rightarrow \infty$ and $t \rightarrow T$, by Fatou, we obtain $\mathbb{E}\left[\left(X_{\tau}\right)^{l}\right] \leq M(l)$ for each stopping time $\tau \in \mathcal{T}$.

We can finish the proof considering $l=q$ for item (i) and $l=r$ for item (ii), and also using the upper bound $f(t, x) \leq C(1+x)$ as well as Assumption (HU) part (ii).

Remark 3.3 In case $U(0)=-\infty$, we can follow the arguments in the Proof of Lemma 3.4 for the case $\pi=1$ and $l=r$ (taking into account that $X_{t}=X_{0} S_{t}$ for $0 \leq t<T$ ) to conclude that

$$
\mathbb{E}\left[\left(S_{t}\right)^{r}\right]=\mathbb{E}\left[\left(1+Z_{0, t}\right)^{r}\right] \leq C(0)<\infty \text { for } 0 \leq t<T .
$$

(we assumed that $S_{0}=1$ above, and we also used that the times $0 \leq t<T$, because are deterministic, belong to $\mathcal{T}$ ). The same argument actually works if we start at any time $0 \leq t<T$, so we have

$$
\mathbb{E}\left[\left(1+Z_{t, s}\right)^{r}\right]=\int_{(-1, \infty)}(1+z)^{r} p(t, s, d z) \leq C(t)<\infty, \text { for } t \leq s<T .
$$

\subsection{Construction of a solution for the DPE}

We provide a constructive proof for the existence of a solution of (3.6) using an iteration scheme. Let us define inductively the sequence of functions $\left(v_{m}\right)_{m}$ in $\mathcal{C}_{U}([0, T) \times(0, \infty))$ by:

$$
v_{0}=U, \quad v_{m+1}=\mathcal{L} v_{m}, \quad m \geq 0 .
$$

Lemma 3.5 Assume (HL), (NA), and (HU). Then the sequence of functions $v_{m}$ satisfies

$$
v_{m} \leq v_{m+1} \leq f, \quad m \geq 0 .
$$

Proof. We do the proof by induction. We obviously have $U=v_{0} \leq v_{1}$. In addition, since the operator $\mathcal{L}$ is monotone and $U \leq f$ we have

$$
v_{1}=\mathcal{L} U \leq \mathcal{L} f \leq f,
$$

so the statement is true for $m=0$. Assume now the statement is true for $m$. We use again the monotonicity of $\mathcal{L}$ to get

$$
v_{m+2}=\mathcal{L} v_{m+1} \geq \mathcal{L} v_{m}=v_{m+1}, \quad v_{m+2}=\mathcal{L} v_{m+1} \leq \mathcal{L} f \leq f,
$$

so the proof is finished.

Under the conditions of the above Lemma, the nondecreasing sequence $\left(v_{m}\right)_{m}$ converges pointwise, and we may define

$$
v^{*}=\lim _{m \rightarrow \infty} v_{m} \leq f .
$$

We show next that $v^{*}$ satisfies the fixed point DP equation. 
Theorem 3.1 Assume that (HL), (NA), (HU) and (HI) hold. Then, $v^{*}$ is solution to the fixed point DP (3.6).

Proof. Fix $\pi \in[0,1]$. We know by construction that

$$
v_{m+1}(t, x) \geq \int_{t}^{T} \int_{(-1, \infty)} \lambda(s) e^{-\int_{t}^{s} \lambda(u) d u} v_{m}(s, x(1+\pi z)) p(t, s, d z) d s .
$$

If $0 \leq \pi<1$, then $v_{m}(s, x(1+\pi z) \geq U(x(1-\pi))$ so the integral on the right hand side is clearly finite. If $\pi=1$, according to Remark 3.3, the integral on the right hand side is still finite for each $m \geq 0$. Therefore, we can let $m \nearrow \infty$ and use the monotone convergence theorem to obtain

$$
v^{*}(t, x) \geq \int_{t}^{T} \int_{(-1, \infty)} \lambda(s) e^{-\int_{t}^{s} \lambda(u) d u} v^{*}(s, x(1+\pi z)) p(t, s, d z) d s .
$$

Since this happens for each $\pi$, taking the supremum over $\pi$ we get $v^{*} \geq \mathcal{L} v^{*}$. Conversely, for $\varepsilon>0$ there exists $m$ such that $v^{*}(t, x)-\varepsilon \leq v_{m+1}(t, x)$ and (because of convexity the maximum is attained) $\pi^{m}(t, x)$ such that

$$
v_{m+1}(t, x)=\int_{t}^{T} \int_{(-1, \infty)} \lambda(s) e^{-\int_{t}^{s} \lambda(u) d u} v_{m}\left(s, x\left(1+\pi^{m}(t, x) z\right)\right) p(t, s, d z) d s .
$$

Since $v_{m} \leq v^{*}$ it follows that

$$
\begin{aligned}
v^{*}(t, x)-\varepsilon & \leq \int_{t}^{T} \int_{(-1, \infty)} \lambda(s) e^{-\int_{t}^{s} \lambda(u) d u} v^{*}\left(s, x\left(1+\pi^{m}(t, x) z\right)\right) p(t, s, d z) d s \\
& \leq \mathcal{L} v^{*}(t, x) .
\end{aligned}
$$

Letting $\varepsilon \rightarrow 0$ we obtain $v^{*}=\mathcal{L} v^{*}$. Finally, since $U(t, x) \leq v^{*}(t, x) \leq f(t, x)$ and the function $f$ satisfies the boundary condition (3.3) by Lemma 3.3. we conclude that $v^{*}$ is a solution to the fixed point DP equation (3.6).

Remark 3.4 The previous theorem shows the existence of a fixed point to the DP equation (3.6), and gives also an iterative procedure for constructing a fixed point. In the next subsection, we shall prove that such a fixed point is equal to the value function $v$, which implies in particular the uniqueness for the fixed point equation (3.6).

\subsection{Verification and optimal strategies}

Consider the solution $v^{*}$ to the fixed point DP equation (3.6), constructed in Theorem 3.1 We now state a verification theorem for the fixed point equation (3.6), which provides the optimal portfolio strategy in feedback form.

Theorem 3.2 Assume that (HL), (NA), (HU) and (HI) hold. Then,

$$
V_{0}=v^{*}\left(0, X_{0}\right)
$$


and an optimal control $\hat{\alpha} \in \mathcal{A}$ is given by

$$
\hat{\alpha}_{n}=\hat{\pi}\left(\tau_{n}, \hat{X}_{\tau_{n}}\right) \hat{X}_{\tau_{n}}, \quad n \geq 0,
$$

where $\hat{\pi}$ is a measurable function on $[0, T) \times(0, \infty)$ solution to

$$
\hat{\pi}(t, x) \in \arg \max _{\pi \in[0,1]} \int_{t}^{T} \int_{(-1, \infty)} \lambda(s) e^{-\int_{t}^{s} \lambda(u) d u} v^{*}(s, x(1+\pi z)) p(t, s, d z) d s,
$$

and $\left(\hat{X}_{\tau_{n}}\right)_{n \geq 0}$ is the wealth given by

$$
\hat{X}_{\tau_{n+1}}=\hat{X}_{\tau_{n}}+\hat{\alpha}_{n} Z_{n+1}, \quad n \geq 0,
$$

and starting from $\hat{X}_{0}=X_{0}$.

Proof. Consider $\alpha \in \mathcal{A}$ and the corresponding positive wealth process $\left(X_{\tau_{n}}\right)_{n \geq 0}$. From Lemma 3.4, we know that

$$
\mathbb{E}\left[\left|v^{*}\left(\tau_{n}, X_{\tau_{n}}\right)\right|\right]<\infty, \quad(\forall) n \geq 0
$$

We apply Lemma 3.1 to get for any $n \geq 0$ :

$$
\begin{aligned}
\mathbb{E}\left[v^{*}\left(\tau_{n+1}, X_{\tau_{n+1}}\right) \mid \mathcal{F}_{n}\right] & =\int_{\tau_{n}}^{T} \int_{(-1, \infty)} \lambda(s) e^{-\int_{\tau_{n}}^{s} \lambda(u) d u} v^{*}\left(s, X_{\tau_{n}}+\alpha_{n} z\right) p\left(\tau_{n}, s, d z\right) d s \\
& \leq \mathcal{L} v^{*}\left(\tau_{n}, X_{\tau_{n}}\right)=v^{*}\left(\tau_{n}, X_{\tau_{n}}\right),
\end{aligned}
$$

so the process $\left\{v^{*}\left(\tau_{n}, X_{\tau_{n}}\right), n \geq 0\right\}$ is a $(\mathbb{P}, \mathbb{F})$-supermartingale. Recalling that $v^{*}(t,) \geq$. we obtain

$$
\mathbb{E}\left[U\left(X_{\tau_{n}}\right)\right] \leq \mathbb{E}\left[v^{*}\left(\tau_{n}, X_{\tau_{n}}\right)\right] \leq v^{*}\left(0, X_{0}\right), \quad(\forall) n \geq 0 .
$$

Now, by Lemma 3.4, the sequence $\left(U\left(X_{\tau_{n}}\right)\right)_{n}$ is uniformly integrable. By sending $n$ to infinity into the last inequality, we then get

$$
\mathbb{E}\left[U\left(X_{T}\right)\right] \leq v^{*}\left(0, X_{0}\right)
$$

Since $\alpha$ is arbitrary, we obtain $V_{0} \leq v^{*}\left(0, X_{0}\right)$.

Conversely, let $\hat{\alpha} \in \mathcal{A}$ be the portfolio strategy given by (3.18), and $\left(\hat{X}_{\tau_{n}}\right)_{n \geq 0}$ the associated wealth process. Then, by the same calculations as in (3.19), we have now the equalities:

$$
\begin{aligned}
\mathbb{E}\left[v^{*}\left(\tau_{n+1}, \hat{X}_{\tau_{n+1}}\right) \mid \mathcal{F}_{n}\right] & =\int_{\tau_{n}}^{T} \int_{(-1, \infty)} \lambda(s) e^{-\int_{\tau_{n}}^{s} \lambda(u) d u} v^{*}\left(s, X_{\tau_{n}}+\hat{\alpha}_{n} z\right) p\left(\tau_{n}, s, d z\right) d s \\
& =\mathcal{L} v^{*}\left(\tau_{n}, \hat{X}_{\tau_{n}}\right)=v^{*}\left(\tau_{n}, \hat{X}_{\tau_{n}}\right), \quad n \geq 0,
\end{aligned}
$$

by definition of $\mathcal{L}$ and $\hat{\alpha}$. This means that the process $\left\{v^{*}\left(\tau_{n}, \hat{X}_{\tau_{n}}\right), n \geq 0\right\}$ is a $(\mathbb{P}, \mathbb{F})$ martingale, and so:

$$
\mathbb{E}\left[v^{*}\left(\tau_{n}, \hat{X}_{\tau_{n}}\right)\right]=v^{*}\left(0, X_{0}\right), \quad(\forall) n \geq 0
$$


From the the bounds $U \leq v^{*} \leq f$ and Lemma 3.4, we know that the sequence $\left(v^{*}\left(\tau_{n}, \hat{X}_{\tau_{n}}\right)\right)_{n}$ is uniformly integrable. By sending $n$ to infinity into the last equality, and recalling the terminal condition for $v^{*}$, we then get

$$
\mathbb{E}\left[U\left(\hat{X}_{T}\right)\right]=v^{*}\left(0, X_{0}\right)
$$

Together with the inequality, $V_{0} \leq v^{*}\left(0, X_{0}\right)$, this proves that $V_{0}=v^{*}\left(0, X_{0}\right)$ and $\hat{\alpha}$ is an optimal control.

An identical verification argument to the proof of Theorem 3.2 can be performed for an investor starting at time $t$ with initial capital $x$ : this way we prove that $v^{*}$ is actually the value function of the control problem. In addition, this shows that the Dynamic Programming Equation (3.6) has a unique solution. For the sake of avoiding the heavy notation associated with strategies starting at time $t$, we decided to only do the verification for time $t=0$.

The Proposition below shows that actually we can approximate the optimal control, and not only the maximal expected utility, using a finite number of iterations. The approximate optimal control is actually very simple, since after the $m$-th arrival time all the wealth is invested in the money market. In addition, a stochastic control representation for the iteration $v_{m}$ is provided.

Proposition 3.1 Assume that (HL), (NA), (HU) and (HI) hold. Then

$$
v_{m}\left(0, X_{0}\right)=\sup _{\alpha \in \mathcal{A}_{m}} \mathbb{E}\left[U\left(X_{T}\right)\right]
$$

where $\mathcal{A}_{m}$ is the set of admissible controls $\alpha=\left(\alpha_{n}\right)_{\geq 0} \in \mathcal{A}$ such that all money is invested in the money market after $m$ arrivals, i.e. $\alpha_{n}=0$ for $n \geq m$.

For any $0 \leq n \leq m-1$, consider the measurable function $\hat{\pi}^{n}(\cdot, \cdot)$ defined by

$$
\hat{\pi}^{n}(t, x)=\arg \max _{\pi \in[0,1]} \int_{t}^{T} \int_{(-1, \infty)} \lambda(s) e^{-\int_{t}^{s} \lambda(u) d u} v_{m-n-1}(s, x(1+\pi z)) p(t, s, d z) d s,
$$

so that

$$
v_{m-n}(t, x)=\int_{t}^{T} \int_{(-1, \infty)} \lambda(s) e^{-\int_{t}^{s} \lambda(u) d u} v_{m-n-1}\left(s, x\left(1+\pi^{n}(t, x) z\right)\right) p(t, s, d z) d s .
$$

Define in feedback form the admissible strategy $\hat{\alpha}^{m} \in \mathcal{A}_{m}$ by $\alpha_{n}^{m}=\hat{\pi}^{n}\left(\tau_{n}, \hat{X}_{\tau_{n}}^{m}\right) \hat{X}_{\tau_{n}}^{m}$ for $0 \leq n \leq m-1$ and $\alpha_{n}^{m}=0$ for $n \geq m$, where the wealth processes $\left(\hat{X}_{\tau_{n}}^{m}\right)_{n \geq 0}$ is given by

$$
\hat{X}_{\tau_{n+1}}^{m}=\hat{X}_{\tau_{n}}^{m}+\hat{\alpha}_{n}^{m} Z_{n+1}, \quad 0 \leq n \leq m-1, \quad \hat{X}_{\tau_{n}}^{m}=\hat{X}_{\tau_{m}}^{m}, \quad n \geq m,
$$

starting from the initial wealth $X_{0}$. Then $\alpha^{m} \in \mathcal{A}_{m}$ is an optimal control for (3.20).

Proof. The proof is based on similar arguments to the proof of Theorem 3.2. Namely, for each $\alpha \in \mathcal{A}_{m}$, one can use Lemma 3.1 to conclude that $\left(v_{m-n}\left(\tau_{n}, X_{n}\right)\right)_{n=0,1, \ldots, m}$ is a supermartingale and, for the particular choice of the control $\alpha^{m}$ described above we actually have that $\left(v_{m-n}\left(\tau_{n}, \hat{X}_{n}^{m}\right)\right)_{n=0,1, \ldots, m}$ is a true martingale. Since $v_{0}(t, x)=U(x)$ and for each 
$\alpha \in \mathcal{A}_{m}$ the wealth process $X$ is constant after the arrival time $\tau_{m}$, it is easy to finish the proof.

Theorems 3.1 and 3.2 together show how we can compute by iterations the maximal expected utility and the optimal control. Since the control problem is finite-horizon in time and infinite horizon in $n$, taking into account Proposition 3.1, the iteration procedure represents exactly the approximation of the infinite horizon problem by a sequence of finite horizon problems.

\subsection{Example: the case of CRRA utility functions}

In this subsection, we consider the case of CRRA (Constant Relative Risk Aversion) utility functions.

First, let us take logarithm utility functions:

$$
U(x)=\ln x, \quad x>0 .
$$

We easily see that in this case the value function has the form:

$$
v(t, x)=U(x)+\varphi(t),
$$

for some nonnegative continuous function $\varphi$ on $[0, T)$ with $\varphi\left(T^{-}\right)=0$. The computation of $\mathcal{L} v$ in (3.5) is straightforward:

$$
\begin{aligned}
\mathcal{L} v=\ln x & +\int_{t}^{T} \lambda(s) e^{-\int_{t}^{s} \lambda(u) d u} \varphi(s) d s \\
& +\sup _{\pi \in[0,1]} \int_{t}^{T} \lambda(s) e^{-\int_{t}^{s} \lambda(u) d u}\left(\int_{(-1, \infty)} \ln (1+\pi z) p(t, s, d z)\right) d s .
\end{aligned}
$$

Hence, we see that $v$ is a solution to the fixed point equation: $\mathcal{L} v=v$ iff $\varphi$ satisfies:

$$
\varphi(t)=\int_{t}^{T} \lambda(s) e^{-\int_{t}^{s} \lambda(u) d u} \varphi(s) d s+F(t), \quad t \in[0, T),
$$

where $F$ is the function defined on $[0, T)$ by

$$
F(t)=\sup _{\pi \in[0,1]} \int_{t}^{T} \lambda(s) e^{-\int_{t}^{s} \lambda(u) d u}\left(\int_{(-1, \infty)} \ln (1+\pi z) p(t, s, d z)\right) d s .
$$

We next consider power utility functions:

$$
U(x)=\frac{x^{\gamma}}{\gamma}, \quad x>0, \gamma<1, \gamma \neq 0
$$

In this case, the value function has the form:

$$
v(t, x)=\varphi(t) U(x),
$$

for some continuous function $\varphi$ on $[0, T)$, greater than 1 , with $\varphi\left(T^{-}\right)=1$. We easily compute $\mathcal{L} v$ in $(3.5)$ :

$$
\mathcal{L} v=U(x) \sup _{\pi \in[0,1]} \int_{t}^{T} \lambda(s) e^{-\int_{t}^{s} \lambda(u) d u} \varphi(s)\left(\int_{(-1, \infty)}(1+\pi z)^{\gamma} p(t, s, d z)\right) d s .
$$


Hence, we see that $v$ is a solution to the fixed point equation: $\mathcal{L} v=v$ iff $\varphi$ satisfies:

$$
\varphi(t)=\sup _{\pi \in[0,1]} \int_{t}^{T} \lambda(s) e^{-\int_{t}^{s} \lambda(u) d u} \varphi(s)\left(\int_{(-1, \infty)}(1+\pi z)^{\gamma} p(t, s, d z)\right) d s, \quad t \in[0, T) .
$$

\section{Convergence in the illiquid market model}

So far, we have considered the optimal investment problem (3.1) for a fixed arrival rate function $\lambda:[0, T) \rightarrow[0, \infty)$ satisfying condition (2.4). To emphasize the dependence on the arrival rate, let us denote by $V_{0}^{\lambda}$ the value in (3.1). When the arrival rate is very large at all times (in some sense to be precised), one would expect that $V_{0}^{\lambda}$ is very close to the optimal expected utility of an agent who can trade at all times (therefore continuously) in the asset $S$. It is also expected that the constraint (2.10), which is implicitly contained in the admissibility condition (2.9) in the discrete time illiquid case, becomes an explicit no-short constraint (2.13) in the continuous time limit. This section is devoted to proving that this is actually true.

First, we need to define the optimization problem for the agent who can trade continuously. We remind the reader that continuous time trading strategies can be defined by (2.12). We denote by $\mathcal{X}^{S}$ the set of positive wealth processes $\left(X_{t}\right)_{0 \leq t \leq T}$ given by (2.12), by using $\mathbb{G}^{S}$-predictable and $S$-integrable processes $H$ satisfying the no-short sale constraint (2.13). The filtration $\mathbb{G}^{S}=\left(\mathcal{G}_{t}^{S}\right)_{0 \leq t \leq T}$ is defined by

$$
\mathcal{G}_{t}^{S}=\sigma\left\{S_{s}, 0 \leq s \leq t\right\} \vee \mathcal{N}
$$

and represents the information one can get from following the asset $S$. Because of the Lévy structure of $S, \mathbb{G}^{S}$ satisfies the usual conditions. We also denote by $\mathcal{D}(S)$ the set of all $\mathbb{G}^{S}$-predictable processes $\pi$ valued in $[0,1]$. It is then clear that

$$
\mathcal{X}^{S}=\left\{X^{(\pi)} \mid \pi \in \mathcal{D}(S)\right\} .
$$

The optimization problem for an agent trading continuously, under no-short selling constraints can be formulated as

$$
V_{0}^{M}=\sup _{X \in \mathcal{X}^{S}} \mathbb{E}\left[U\left(X_{T}\right)\right]=\sup _{\pi \in \mathcal{D}(S)} \mathbb{E}\left[U\left(X_{T}^{(\pi)}\right)\right]
$$

The main result of this section is:

Theorem 4.1 Under Assumptions (HL), (NA), (HU) and (HI), consider $\left(\lambda_{k}\right)_{k}$ a sequence of intensity functions satisfying (2.4). If

$$
\sum_{k=0}^{\infty} \exp \left(-\int_{t}^{s} \lambda_{k}(u) d u\right)<\infty, \quad(\forall) \quad 0 \leq t<s<T,
$$

then

$$
V_{0}^{\lambda_{k}} \rightarrow V_{0}^{M}, \quad \text { as } k \text { goes to infinity, }
$$

where $V_{0}^{M}$ is defined by (4.1). 
Remark 4.1 Condition (4.2) is satisfied for example with $\lambda_{k}(t)=k \cdot \lambda(t)$, where $\lambda$ is an intensity function satisfying (2.4). This condition also implies that for all $0 \leq t<s<T$,

$$
\int_{t}^{s} \lambda_{k}(u) d u \rightarrow \infty, \quad \text { as } k \text { goes to infinity. }
$$

In order to prove Theorem 4.1, we first have to put all the optimization problems (3.1) on the same physical probability space, independent of the intensity function $\lambda$. This is an easy task actually. We consider a probability space $(\Omega, \mathcal{G}, \mathbb{P})$ supporting two independent processes: the continuous time stock price process $\left(S_{t}\right)_{0 \leq t \leq T}$ (which has all the desired properties) and a Poisson process $\left(M_{t}\right)_{0 \leq t<\infty}$ with intensity equal to one. After that, for each intensity function $\lambda$ we define the nonhomogenous Poisson process $N^{\lambda}$ (actually its sequence of jumps) by (2.5). Therefore, for different intensities, we still have the same physical space. We now denote by $\mathbb{F}^{\lambda}$ and $\mathbb{G}^{\lambda}$ the discrete and continuous time filtrations on $(\Omega, \mathcal{G}, \mathbb{P})$ defined by (2.7) and (2.11) corresponding to the intensity $\lambda$, and by $\tau_{n}^{\lambda}$ the associated jump times.

The main obstacle in proving Theorem 4.1 is the fact that the filtration $\mathbb{F}^{\lambda}$ only observes the process $S$ at the arrival times, while the filtration $\mathbb{G}^{S}$ used by the investor in (4.1) observes the stock continuously. This problem is overcome in three steps

Step 1: first, we show that in (3.1), the discrete-time filtration $\mathbb{F}^{\lambda}=\left(\mathcal{F}_{n}^{\lambda}\right)_{n \geq 0}$ can be replaced by the larger filtration $\left(\mathcal{G}_{\tau_{n}^{\lambda}}^{\lambda}\right)_{n \geq 0}$. In other words, due to the Markov structure of the model, an investor who can only trade at the discrete arrival times, cannot improve his/her expected utility by continuously observing the evolution of the stock between the arrival times. This is done in Lemma 4.1 below.

Lemma 4.1 Fix an intensity function $\lambda$ and define

$$
V_{0}^{\lambda, c}:=\sup _{\alpha \in \mathcal{A}_{c}^{\lambda}} \mathbb{E}\left[U\left(X_{T}\right)\right]
$$

where $\mathcal{A}_{c}^{\lambda}$ is the set of simple admissible strategies $\alpha=\left(\alpha_{n}\right)_{n \geq 0}$ with continuous observation, which means that for each $n \geq 0$ we have $\alpha_{n} \in \mathcal{G}_{\tau_{n}^{\lambda}}^{\lambda}$ and $\alpha$ satisfies the constraint (2.10) for the wealth process $\left(X_{\tau_{n}}\right)_{n \geq 0}$ defined by (2.8).

Then, under Assumptions (HL), (NA), (HU) and (HI), we have $V_{0}^{\lambda, c}=V_{0}^{\lambda}$.

Proof. Similarly to Remark 2.2. Assumption (HL) together with the independence of $S$ and $N$ ensures that for all $n \geq 0$, the (regular) distribution of $\left(\tau_{n+1}, Z_{n+1}\right)$ conditioned on $\mathcal{G}_{\tau_{n}^{\lambda}}^{\lambda}$ is given by:

1. $\mathbb{P}\left[\tau_{n+1}^{\lambda} \in d s \mid \mathcal{G}_{\tau_{n}^{\lambda}}^{\lambda}\right]=\lambda(s) e^{-\int_{\tau_{n}}^{s} \lambda(u) d u} d s$

2. further conditioning on knowing the next arrival time $\tau_{n+1}^{\lambda}$, the return $Z_{n+1}$ has distribution

$$
\mathbb{P}\left[Z_{n+1} \in d z \mid \mathcal{G}_{\tau_{n}^{\lambda}}^{\lambda} \vee \sigma\left(\tau_{n+1}^{\lambda}\right)\right]=p\left(\tau_{n}^{\lambda}, \tau_{n+1}^{\lambda}, d z\right)
$$


Therefore, in Lemma 3.1, one can replace the filtration $\mathbb{F}^{\lambda}$ by the larger filtration $\left(\mathcal{G}_{\tau_{n}^{\lambda}}^{\lambda}\right)_{n \geq 0}$ and obtain that, for each $\alpha \in \mathcal{A}_{c}^{\lambda}$ we have

$$
\mathbb{E}\left[v\left(\tau_{n+1}^{\lambda}, X_{\tau_{n+1}^{\lambda}}\right) \mid \mathcal{G}_{\tau_{n}^{\lambda}}^{\lambda}\right]=\int_{\tau_{n}^{\lambda}}^{T} \int_{(-1, \infty)} \lambda(s) e^{-\int_{\tau_{n}^{\lambda}}^{s} \lambda(u) d u} v\left(s, X_{\tau_{n}^{\lambda}}+\alpha_{n} z\right) p\left(\tau_{n}^{\lambda}, s, d z\right) d s .
$$

After that, one can just follow the verification arguments in the proof of Theorem 3.2 to show that

$$
V_{0}^{\lambda, c}=v^{*, \lambda}\left(0, X_{0}\right)
$$

which ends the proof.

Step 2: we define the continuous time filtration $\mathbb{G}^{\infty}=\left(\mathcal{G}_{t}^{\infty}\right)_{0 \leq t \leq T}$ which contains all the information from the arrival times right at time zero. This corresponds to an investor who knows in advance all the jumps of the homogeneous Poisson process $M$ and also observes continuously the stock $S$ up to time $t$ :

$$
\mathcal{G}_{t}^{\infty}=\mathcal{G}_{t}^{S} \vee \sigma\left(M_{u}: 0 \leq u<\infty\right), \quad 0 \leq t<T .
$$

Because the information added is independent, the process $S$ is still a semimartingale with respect to the larger filtration $\mathbb{G}^{\infty}$, which satisfies the usual conditions as well. Using again the independence property, Lemma 4.2 below shows that if the investor in (4.1) has the additional information in $\mathbb{G}^{\infty}$, he/she cannot improve the maximal expected utility.

Lemma 4.2 Consider the set $\overline{\mathcal{X}}_{c}$ of wealth processes defined by (2.12) where the general integrand $H$ is $\mathbb{G}^{\infty}$-predictable, $S$-integrable and satisfies (2.13). Define

$$
V_{0}^{\infty}:=\sup _{X \in \overline{\mathcal{X}}_{c}} \mathbb{E}\left[U\left(X_{T}\right)\right]
$$

Then $V_{0}^{\infty}=V_{0}^{M}$.

Proof. Since $\mathcal{X}^{S} \subset \overline{\mathcal{X}}_{c}$, we obviously have $V_{0}^{M} \leq V_{0}^{\infty}$. Now take some arbitrary $X \in \overline{\mathcal{X}}_{c}$ associated to a no-short sale trading strategy $H$, which is $\mathbb{G}^{\infty}$-predictable. Consider the $\mathbb{G}^{S}$-predictable projection of $H: \hat{H}_{s}=\mathbb{E}\left[H_{s} \mid \mathcal{G}_{s^{-}}^{S}\right], t \leq s \leq T$. We then have

$$
\hat{X}_{t}:=\mathbb{E}\left[X_{t} \mid \mathcal{G}_{t}^{S}\right]=X_{0}+\int_{0}^{t} \hat{H}_{u} d S_{u}, \quad 0 \leq t \leq T
$$

This means that the process $\hat{X}$ lies in $\mathcal{X}^{S}$. Since $U$ is concave, we get by the law of iterated conditional expectations and Jensen's inequality

$$
\mathbb{E}\left[U\left(X_{T}\right)\right]=\mathbb{E}\left[\mathbb{E}\left[U\left(X_{T}\right) \mid \mathcal{G}_{T}^{S}\right]\right] \leq \mathbb{E}\left[U\left(E\left[X_{T} \mid \mathcal{G}_{T}^{S}\right]\right)\right]=\mathbb{E}\left[U\left(\hat{X}_{T}\right)\right] \leq V_{0}^{M}
$$

We conclude from the arbitrariness of $X$ in $\overline{\mathcal{X}}_{c}$.

Step 3: Once we prove the step above and transform the Merton problem in a utility maximization problem with no short-sale constraints under the filtration $\mathbb{G}^{\infty}$, we can basically follow the arguments in Theorems 3.1, 3.3 and 4.1 in [4], to finish the proof. 
Proof of Theorem 4.1. We first represent continuous time trading strategies with no short sale constraints in terms of proportion of wealth, for the larger filtration $\mathbb{G}^{\infty}$. For any continuous time wealth process $X \in \overline{\mathcal{X}}_{c}$ associated to a trading strategy $H$ satisfying (2.13), we still denote by $\pi_{t}=H_{t} S_{t^{-}} / X_{t-}$, and notice that the process $\left(\pi_{t}\right)_{0 \leq t \leq T}$ is valued in $[0,1]$ by (2.13). We also denote by $X^{(\pi)}$ the process defined by (2.14) and define $\mathcal{D}^{\infty}$ to be the set of all $\mathbb{G}^{\infty}$-predictable processes $\pi$ valued in $[0,1]$. It is then clear that

$$
V_{0}^{\infty}=\sup _{\pi \in \mathcal{D}^{\infty}} \mathbb{E}\left[U\left(X_{T}^{(\pi)}\right)\right]
$$

Using Lemma 4.1 and Lemma 4.2 we have, for each intensity function $\lambda$ that

$$
V_{0}^{\lambda}=V_{0}^{\lambda, c} \leq V_{0}^{\infty}=V_{0}^{M} .
$$

Let now $\pi \in \mathcal{D}(S) \subset \mathcal{D}^{\infty}$ be a (proportional) trading strategy in (4.1) and let $\left(\lambda_{k}\right)_{k}$ a sequence of intensity functions as in Theorem 4.1. We will follow the arguments in [4] to approximate this continuous time trading strategy by simple strategies $\alpha^{k} \in \mathcal{A}_{c}^{\lambda_{k}}$, which are discrete, but use information from continuous observations. First, according to Lemma 3.4 and 3.5 in [4], there exists a sequence $\pi^{m} \in \mathcal{D}(S)$ such that each $\pi^{m}$ is LCRL (left continuous with right limits) and such that

$$
u c \mathbb{P}-\lim _{m \rightarrow \infty} X^{\left(\pi^{m}\right)}=X^{(\pi)},
$$

so, in order to approximate $\pi$ we can actually assume it is LCRL. Here, by $u c \mathbb{P}$-convergence, we mean the usual convergence of processes in probability, uniformly on compact time-sets.

Let us check that our sequence of stopping times satisfies the condition $\sup _{n}\left|\tau_{n+1}^{k}-\tau_{n}^{k}\right| \rightarrow$ 0 a.s., $k \rightarrow \infty$. Take a subdivision $0=t_{0}<\ldots<t_{i}<\ldots<t_{M}=T$ of $[0, T]$ such that $\left|t_{i+1}-t_{i}\right| \leq \epsilon / 2$, for all $i$. We then have :

$$
\begin{aligned}
\mathbb{P}\left[\sup _{n}\left|\tau_{n+1}^{k}-\tau_{n}^{k}\right|>\epsilon\right] & \leq \sum_{i=0}^{M-1} \mathbb{P}\left[\exists n, \tau_{n}^{k} \leq t_{i}<t_{i+1} \leq \tau_{n+1}^{k}\right] \\
& =\sum_{i=0}^{M-1} \mathbb{P}\left[N_{t_{i}}^{\lambda_{k}}=N_{t_{i+1}}^{\lambda_{k}}\right] \\
& =\sum_{i=0}^{M-1} \exp \left(-\int_{t_{i}}^{t_{i+1}} \lambda_{k}(u) d u\right) .
\end{aligned}
$$

By Borel-Cantelli and (4.2), we deduce that

$$
\mathbb{P}\left[\limsup _{k} \sup _{n}\left|\tau_{n+1}^{k}-\tau_{n}^{k}\right|>\epsilon\right]=0, \quad(\forall) \varepsilon>0 .
$$

Next, let us define

$$
\pi_{n}^{k}=\pi_{\tau_{n}^{\lambda^{k}}+}, \quad n \geq 0,
$$

where $\pi_{t+}=\lim _{u \unlhd t} \pi_{t}$. Because the filtration $\mathbb{G}^{\lambda^{k}}$ satisfies the usual conditions we have that the process $\left(\pi_{t+}\right)_{0 \leq t \leq T}$ is optional with respect to $\mathbb{G}^{\lambda_{k}}$ for each $k$. Since, in addition, $\tau_{n}^{\lambda_{k}}$ are stopping times with respect to $\mathbb{G}^{\lambda_{k}}$ we obtain that, for each $k$,

$$
\pi_{n}^{k} \in \mathcal{G}_{\tau_{n}^{\lambda_{k}}}^{\lambda^{k}}, \quad k \geq 0 .
$$


Therefore, if we define, for each fixed $k$ the discrete-time wealth process by

$$
X_{\tau_{n+1}^{\lambda_{k}}}=X_{\tau_{n}^{\lambda_{k}}}\left(1+\pi_{n}^{k} Z_{n+1}\right), \quad n \geq 0
$$

and denote by $\alpha_{n}^{k}=\pi_{n}^{k} X_{\tau_{n}^{\lambda_{k}}}$ we have $\alpha^{k}=\left(\alpha_{n}^{k}\right)_{n \geq 0} \in \mathcal{A}_{c}^{\lambda_{k}}$. To each of the above defined $\alpha^{k}$, we can associate by Remark 2.5 a continuous time simple integrand $H^{k}$ which is $\mathbb{G}^{\lambda_{k}}$-predictable and the continuous time wealth process $\left(X_{t}^{k}\right)_{0 \leq t \leq T}$. The fundamental observation is now that all $H^{k}$ are predictable with respect to the same "large" filtration $\mathbb{G}^{\infty}$. Using this universal filtration, we can now follow the proof of Theorem 3.1 in [4], which actually works for stochastic partitions under condition (4.5), to conclude that

$$
X^{(\pi)}=u c \mathbb{P}-\lim _{k \rightarrow \infty} X^{k}
$$

Therefore, we can approximate any continuous time strategy in the Merton problem (4.1) by simple trading strategies $\alpha^{k} \in \mathcal{A}_{c}^{\lambda_{k}}$. The rather obvious details on how approximation of strategies leads to approximation of optimal expected utility are identical to the arguments in [4] Section 4, and are omitted: this means that for all $\pi \in \mathcal{D}(S), \mathbb{E}\left[U\left(X_{T}^{(\pi)}\right)\right]=$ $\lim _{k} \mathbb{E}\left[U\left(X_{T}^{k}\right)\right]$, and so $V_{0}^{M} \leq \liminf _{k} V_{0}^{\lambda_{k}, c}$. Together with (4.4), this concludes the proof of Theorem 4.1 .

\section{References}

[1] Bertsekas D. and S. Shreve (1978): Stochastic optimal control: the discrete-time case, Academic Press.

[2] Cretarola A., Gozzi F., Pham H. and P. Tankov (2008): "Optimal consumption policies in illiquid markets", to appear in Finance and Stochastics.

[3] Jacod J. and A. Shiryaev (2003): Limit theorems for stochastic processes, 2nd edition, Springer Verlag.

[4] Kardaras, K. and E. Platen (2008): "Multiplicative approximation of wealth processes involving no-short-sale strategies via simple trading", preprint, http://arxiv.org/abs/0812.0033

[5] Matsumoto K. (2006): "Optimal portfolio of low liquid assets with a log-utility function", Finance and Stochastics, 10, 121-145.

[6] Pham H. and P. Tankov (2008): "A model of optimal consumption under liquidity risk with random trading times", Mathematical Finance, 18, 613-627.

[7] Rogers C. and O. Zane (2002): "A simple model of liquidity effects", in Advances in Finance and Stochastics: Essays in Honour of Dieter Sondermann, eds. K. Sandmann and P. Schoenbucher, pp 161-176. 